\title{
Evolutionary origin of gastrulation: insights from sponge development
}

\author{
Nagayasu Nakanishi, Shunsuke Sogabe and Bernard M Degnan*
}

\begin{abstract}
Background: The evolutionary origin of gastrulation-defined as a morphogenetic event that leads to the establishment of germ layers - remains a vexing question. Central to this debate is the evolutionary relationship between the cell layers of sponges (poriferans) and eumetazoan germ layers. Despite considerable attention, it remains unclear whether sponge cell layers undergo progressive fate determination akin to eumetazoan primary germ layer formation during gastrulation.

Results: Here we show by cell-labelling experiments in the demosponge Amphimedon queenslandica that the cell layers established during embryogenesis have no relationship to the cell layers of the juvenile. In addition, juvenile epithelial cells can transdifferentiate into a range of cell types and move between cell layers. Despite the apparent lack of cell layer and fate determination and stability in this sponge, the transcription factor GATA, a highly conserved eumetazoan endomesodermal marker, is expressed consistently in the inner layer of A. queenslandica larvae and juveniles.

Conclusions: Our results are compatible with sponge cell layers not undergoing progressive fate determination and thus not being homologous to eumetazoan germ layers. Nonetheless, the expression of GATA in the sponge inner cell layer suggests a shared ancestry with the eumetazoan endomesoderm, and that the ancestral role of GATA in specifying internalised cells may antedate the origin of germ layers. Together, these results support germ layers and gastrulation evolving early in eumetazoan evolution from pre-existing developmental programs used for the simple patterning of cells in the first multicellular animals.
\end{abstract}

Keywords: Evolution, Gastrulation, Germ layers, Sponge development, GATA

\section{Background}

It has been long argued and widely accepted that early metazoan evolution included the progressive addition and elaboration of cell layers, with the first animals being little more than a small sphere of epithelial cells. The addition of a second inner layer is thought to have followed, resulting in the so-called 'gastraea' that possessed an ectoderm and endoderm. A middle mesodermal layer is proposed to have evolved last $[1,2]$. It has been noted that this progression is reflected in contemporary early branching animals, with sponges and placozoans being interpreted as having one, two or no germ layers, cnidarians and ctenophores possessing two or possibly three germ layers and bilaterians having three or possibly four $[2,3]$. The generation of two or

\footnotetext{
*Correspondence: b.degnan@uq.edu.au

School of Biological Sciences, University of Queensland, Brisbane, QLD 4072, Australia
}

(C) 2014 Nakanishi et al.; licensee BioMed Central Ltd. This is an Open Access article distributed under the terms of the Creative Commons Attribution License (http://creativecommons.org/licenses/by/2.0), which permits unrestricted use, distribution, and reproduction in any medium, provided the original work is properly cited.

more germ layers by gastrulation and the consistency of the fate of these layers unites the Eumetazoa (though exceptions exist; for example, vertebrate tail-bud mesoderm [2]). Further, comparative developmental genetic evidence supports the homology of the endomesoderm [4] and ectodermal neurogenesis [5] across eumetazoans. However, it remains controversial whether the cell layers in sponges are homologous to eumetazoan germ layers and whether sponges undergo gastrulation.

Sponges consist of four classes - Demospongiae, Calcarea, Homoscleromorpha and Hexactinellida [6,7] - of simple animals that share a common body organisation. As juveniles and adults, they are sessile, tri-layered animals that lack true muscles and nerves. Sponges possess an internal network of canals and ciliated choanocyte chambers lined with epithelial cells, primarily endopinacocytes and choanocytes, and are separated from the external environment by another epithelial layer, the exopinacoderm (Figure 1; 


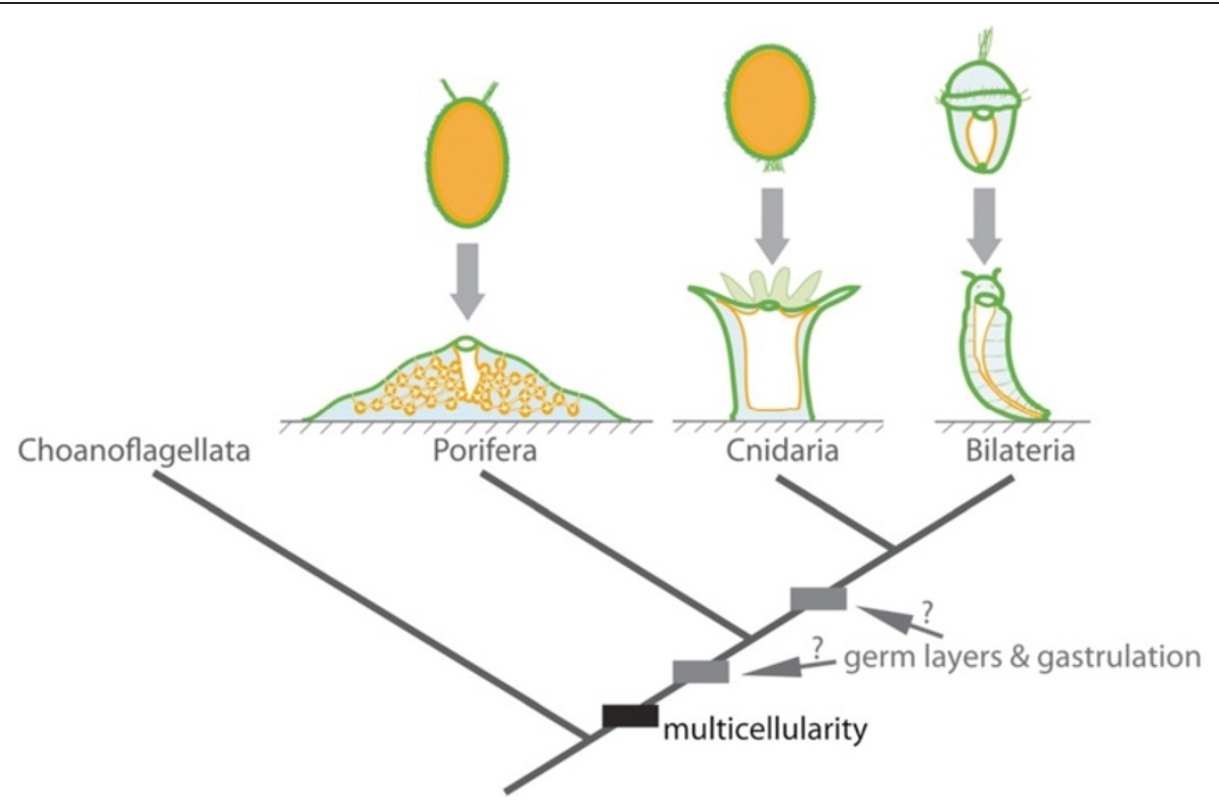

Figure 1 Evolution of germ layers and gastrulation. Animal multicellularity, with the larval and juvenile/adult body plans of extant metazoans possessing multiple cell layers, is depicted above. The arrow represents metamorphosis from larval to juvenile/adult forms. Cell layers are coloured: orange, inner layer; green, outer layer; and blue, middle layer. In the case of cnidarians and bilaterians, these correspond to endoderm, ectoderm and mesoderm (bilaterians only), respectively. The phylogenetic relationship of these clades and the sister group to metazoans, the choanoflagellates, is shown below, along with the evolutionary origin of multicellularity. The origin of germ layers and gastrulation is debatable, occurring either before or after the divergence of sponges and eumetazoans.

hexactinellids are constructed as a syncytium). Sandwiched between these epithelial layers is the collagenous mesohyl, which is enriched with multiple types of amoebocytes, including the pluripotent stem cell type, the archeocyte (Figure 1). Water currents created by ciliary beating in choanocyte chambers enable most of the physiological requirements of sponges, including feeding, respiration, excretion and reproduction. This juvenile/ adult body plan is the outcome of the dramatic reorganisation of the radially-symmetrical, bi- or trilayered larva at metamorphosis [8].

Sponges utilise recognisable gastrulation-like morphogenetic movements during embryogenesis (for example, delamination, ingression, egression and invagination) and metamorphosis (for example, epithelial-mesenchyme transitions, EMT) (summarised in [8,9]). In marine haplosclerid demosponges, for instance, morphological evidence suggests that early cleavage leads to the formation of a stereoblastula, which then delaminates to form a multilayered parenchymella larva (for example, Amphimedon queenslandica [10]). In homoscleromorphs (for example, Oscarella spp.), multipolar egression of a stereoblastula generates a single-layered cinctoblastula larva [11], while in a hexactinillid (for example, Oopsacas minuta), cellular delamination of a coeloblastula gives rise to the trichimella larva $[12,13]$. Interestingly, the pattern of embryogenesis can be polymorphic. In a halisarcid demosponge (for example, Halisarca dujardini), multipolar ingression and/or invagination of a coeloblastula or a stereoblastula forms a multilayered parenchymella or disphaerula ('a hollow sphere within a sphere') larva [14,15]. Epithelialmesenchymal transitions are common at metamorphosis in diverse homoscleromorphs, calcareans and demosponges (reviewed in [8]).

Despite the occurrence of gastrulation-like morphogenetic processes in sponges, it is still unclear how sponge development relates to eumetazoan gastrulation, which is defined as a morphogenetic event that leads to the establishment of germ layers. Some authors consider cell movements that generate multi-layered embryos during sponge embryogenesis gastrulation (for example, [12,16]), although some argue that gastrulation occurs during sponge metamorphosis when an adult internal layer develops by EMT and transdifferentiation of larval ciliated epithelial cells (for example, [17]). Yet it remains unclear whether sponge cell layers display the defining characteristic of eumetazoan gastrulation. This uncertainty led others to question the existence of germ layers, and hence gastrulation, in sponges (for example, [18]).

Here, we show by cell-labelling experiments in the demosponge $A$. queenslandica that the cell layers established during embryogenesis lack fixed identities and do not directly give rise to the cell layers of the juvenile. In addition, inner epithelial choanocytes in the juvenile can alter their fate by transdifferentiating into a range of cell types, including cells of the outer epithelium. Thus, 
sponge cell layers lack fate determination and stability. However, we find that expression of the sponge orthologue to the highly conserved eumetazoan endomesodermal marker GATA [4] is consistently restricted to the inner layer of larvae and juveniles, suggesting a deep shared ancestry between the sponge internal cell layer and the eumetazoan endomesoderm.

\section{Results and discussion}

Germ layers by definition exhibit restricted cell fate $[2,3]$. Thus, to address whether sponge embryonic and early juvenile cell layers are germ layers, we employed cell-labelling techniques in the demosponge $A$. queenslandica to examine the fate of larval epithelial and internal cells at metamorphosis, as well as the stability and fate of differentiated choanocytes in newly established juvenile tissues at the so-called 'rhagon' stage (staging described in Additional file 1: Figure S1).

The swimming A. queenslandica larva metamorphoses into a feeding juvenile with choanocyte chambers within three days of attaching its anterior end onto a natural substratum (crustose coralline algae) at $25^{\circ} \mathrm{C}$. Within hours of settling, the three-layered, bullet-shaped larva transforms into an encrusting mat. A previous celllabelling study in A. queenslandica indicated that external larval cells transdifferentiated into internal choanocytes in juveniles [10]. However, it was not determined whether all cell types found on the larval exterior are transdifferentiating at metamorphosis, and whether the larval epithelial cells differentiate directly into choanocytes or indirectly via an intermediate cell type. In other sponges, the larval epithelial layer has been reported to shed entirely [19], be phagocytised by archeocytes [20-22], differentiate into choanocytes through a nonciliated amoebocyte intermediate [23-25], or directly differentiate into choanocytes without loss of cilia [26] (reviewed in [8]). Using the terminal deoxynucleotidyl transferase dUTP nick end-labelling (TUNEL) assay for detecting apoptotic DNA fragmentation, we demonstrate that a large proportion of A. queenslandica larval epithelial cells undergo programmed cell death shortly after the onset of metamorphosis [see Additional file 2: Figure S2]. TUNEL-labelled nuclear fragments can be observed readily in the metamorphosing animal, often as phagocytised bodies within archeocytes [see Additional file 2: Figure S2].

To study the fate of non-apoptosing larval epithelial cells, the outer layer was labelled with the plasmamembrane dye CM-DiI. We assume that the cell lineage will be indicated by retention of this label in their plasma membrane, while the plasma membrane of the cells that phagocytised apoptotic fragments of larval epithelial origin will not be labelled. An overnight incubation of freeswimming A. queenslandica larvae in seawater containing
$10 \mu \mathrm{M}$ CM-DiI strongly labelled the plasma membrane of ciliated epithelial cell types, the columnar epithelial cell and the flask cell (Figure 2A). During metamorphosis, these labelled cells internalised, resorbing their cilia and differentiating into archeocytes, which migrated throughout the animal [see Additional file 3: Figure S3]. Within two to three days, a number of juvenile cell types were labelled, including choanocytes and exopinacocytes (Figure 2B, C), suggesting that these differentiated from pluripotent archeocytes, which were originally derived from the larval epithelium. Therefore, at metamorphosis ciliated epidermal cells of the larvae can either undergo apoptosis or transdifferentiate, via an archeocyte intermediate, into a range of juvenile cell types that are found in the inner, outer or middle layer of the juvenile.

Using the thymidine analog, 5-ethynyl-2'-deoxyuridine (EdU), to label the nuclei of proliferating cells, we found that only amoeboid cells localised to the inner cell mass, identified here as archeocytes [see Additional file 4: Figure S4], and no other cell types, continue to proliferate in the swimming larva (Figure 2D). Taking advantage of this observation we followed the fate of these EdU-labelled cells during metamorphosis. We found that these archeocytes of the larval inner cell mass origin also gave rise to choanocytes and exopinacocytes during metamorphosis (Figure 2E, F). Together, these observations indicate that a range of larval cell types can generate the diversity of cell types present in the juvenile and that there is no correspondence between the cell layers established during embryogenesis and those produced at metamorphosis.

Sponge metamorphosis has been regarded by some as gastrulation (summarised in $[8,17]$ ). As observed here and in previous studies, morphogenetic processes operational during sponge metamorphosis occur also during eumetazoan gastrulation (for example, EMT; reviewed in $[8,27])$. Therefore, we sought to determine if cell layers established at the end of $A$. queenslandica metamorphosis are developmentally determined. To test this, we labelled choanocytes that form internal choanocyte chambers and a small number of archeocytes surrounding choanocyte chambers by incubating juveniles with CM-DiI (Figure 3A, $\mathrm{B})$. Within one week, the majority of the labelled choanocytes had undergone EMT and dedifferentiated into archeocytes (Figure 3C, D; Additional file 5: Figure S5), of which some had migrated away from the chambers and differentiated into other cell types including exopinacocytes (Figure 3E) and sclerocytes (Figure 3F). Thus, inner epithelial choanocytes are capable of transdifferentiating into outer epithelial cells via an archeocyte intermediate, demonstrating the lack of cell fate determination.

The lack of fate determination in cell layers in $A$. queenslandica is consistent with this demosponge lacking germ layers as classically defined. In light of this 

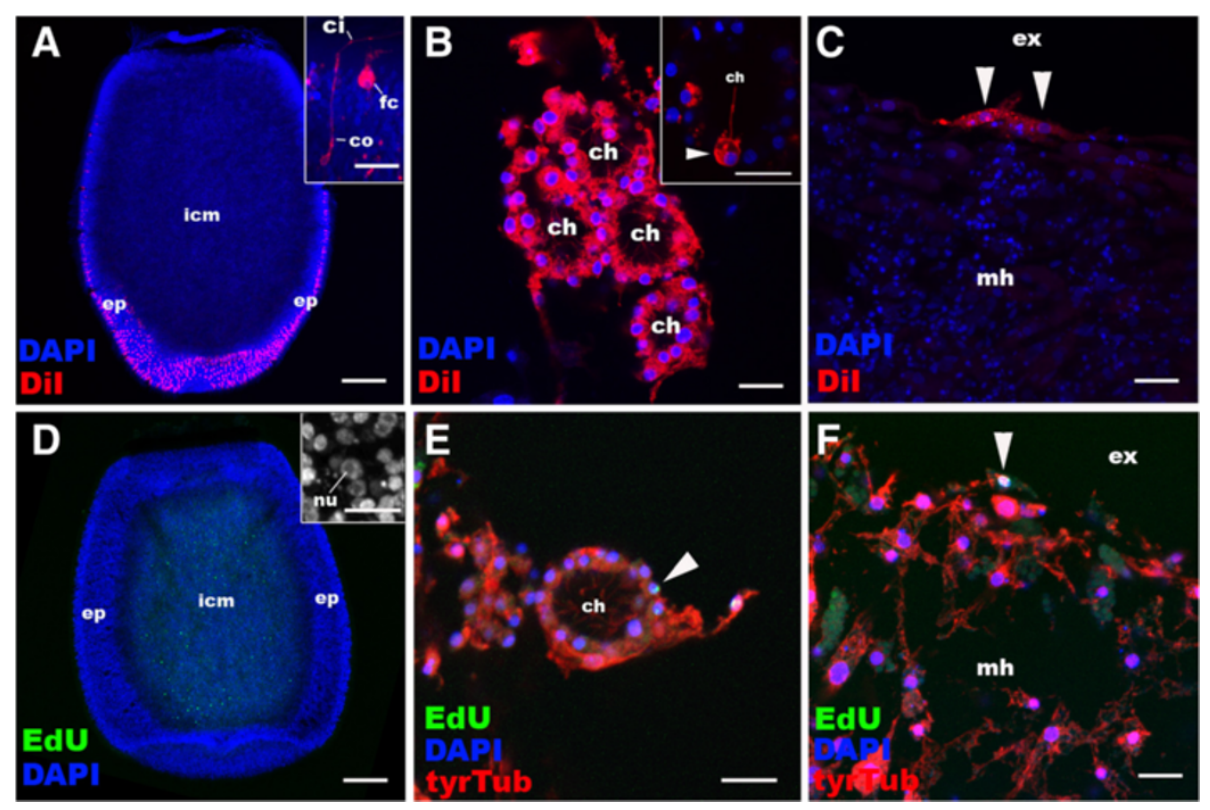

Figure 2 Cell labelling and lineage-tracing through metamorphosis in Amphimedon queenslandica. A, D: Distributions of labelled cells in free-swimming larvae. Anterior (swimming axis) is to the bottom. B, C, E, F: Descendants of the labelled cells in juveniles. Nuclei are stained with DAPI. In $\mathbf{E}$ and $\mathbf{F}$, the juveniles are labelled with an antibody against tyrosinated tubulin (tyrTub). A: Longitudinal confocal sections through the centre of a free-swimming larva incubated with CM-Dil, showing strong labelling in ciliated epidermal cell types, the columnar epithelial cell (co) and the flask cell (fc) (inset), with little labelling in inner cell mass (icm). B: Choanocytes in chambers (ch). In some cases, a subset of choanocytes is CM-Dil-labelled in a single choanocyte chamber (arrowhead in inset), suggesting that multiple precursor cells can be involved in development of a single chamber. C: Labelled exopinacocytes (arrowheads). D: A confocal longitudinal section through the centre of a free-swimming larva pulse-labelled with EdU. Note that the labelled cells localise in the inner cell mass (icm) and are likely to be proliferating archeocytes with characteristic large nucleoli (nu) [see Additional file 3: Figure S3]. E: An EdU-positive choanocyte in the chamber (arrowhead). F: An EdU-positive exopinacocyte (arrowhead). Other abbreviations: ep, outer layer epithelium; ci, cilium; ex external environment; mh, mesohyl. Scale bars: $100 \mu \mathrm{m}$ (A, D), $10 \mu \mathrm{m}$ (B, C, E, F, inset in D), $5 \mu \mathrm{m}$ (inset in A). DAPI, 4',6-diamidino-2-phenylindole; EdU, 5-ethynyl-2'-deoxyuridine.

finding, genes shown to be instrumental in eumetazoan ectoderm or endomesoderm formation may not be expected to be present in demosponges. Indeed, of the genes previously implicated in the differentiation of the endomesoderm across Cnidaria and Bilateria, namely twist, snail, forkhead and GATA [4], only GATA was identified in the genome of the demosponge $A$. queenslandica [28] [see Additional file 6: Figure S6]. Also, amongst the 51 transcription factor- and signalling molecule-encoding genes shown to be 'endomesodermally' expressed in the cnidarian Nematostella vectensis [29], only nine orthologues are present in the A. queenslandica genome [see Additional file 7: Table S1].

A. queenslandica GATA transcripts are localised to a substantial fraction of internal cells of late-stage embryos, free-swimming larvae (Figure 4B-D) and juveniles (Figure 4F-I), but not detectable in outer layer epithelial cells at any developmental stage, similar to predominantly internal (that is, endomesodermal) expression of bilaterian GATA456 [30-32] and cnidarian GATA [4]. Thus, although this study argues against the existence of true germ layers in demosponges, the striking parallel in GATA expression patterns suggests a common ancestry between the sponge inner cell layer and the eumetazoan endomesoderm.

GATA genes are present in other early branching taxa, including placozoans, ctenophores and the other classes of sponges [33-35], although A. queenslandica is the only species for which there currently are published expression patterns. Understanding the evolution of germ layers will be assisted by the analysis of the evolution and expression of genes conserved in bilaterian mesoderm specification and formation, along with further analysis of developmental processes in these taxa. For example, recent analysis of the genome of the ctenophore Mnemiopsis leidyi failed to reveal many 'mesodermal' genes despite it appearing to have genuine endomesoderm [34], and it is known that cell movements from one embryonic layer to another occur during metamorphosis in some medusozoan cnidarians $[36,37]$. These approaches, along with resolving the exact order in which these early-branching metazoans diverged from the lineage leading to bilaterians (that is, whether sponges or ctenophores are the sister group to all other animals and whether sponges are monophyletic $[34,38])$, provide an opportunity to reconstruct the evolution of germ layers and gastrulation. 

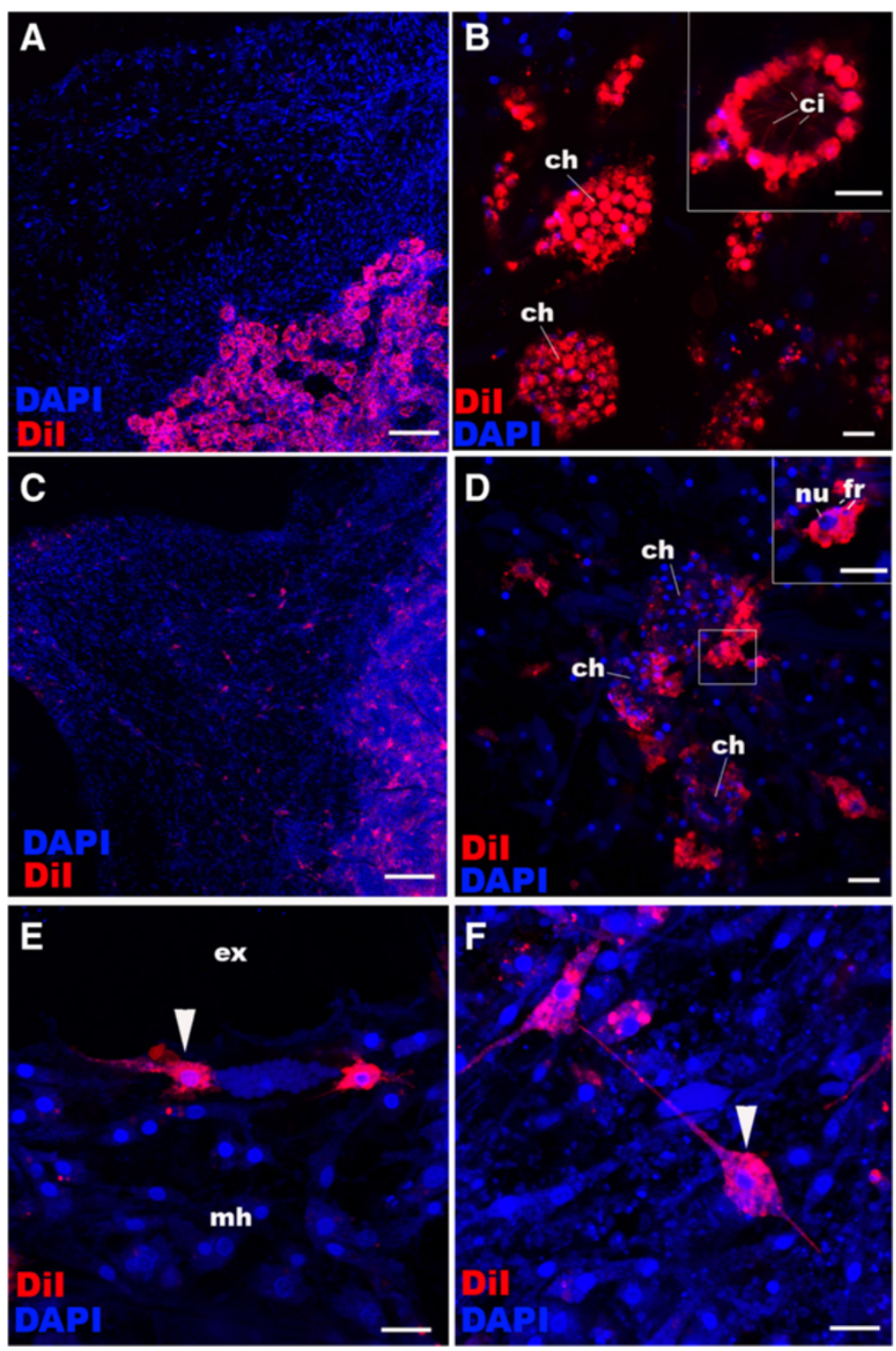

Figure $\mathbf{3}$ Choanocyte labelling and lineage-tracing in juvenile Amphimedon queenslandica. A, B: Choanocytes in chambers (ch) in juveniles are labelled with CM-Dil; note strong plasma membrane labelling in choanocytes (inset). C-F: Descendants of juveniles whose chambers were labelled with CM-Dil seven days earlier (as in A). Multiple other juvenile cell types are now labelled and spread through the sponge body. Note in (D) the limited number of CM-Dil-labelled choanocytes remaining in the choanocyte chambers (ch) seven days after labelling and the presence of labelled archeocytes with large nuclei (nu; inset), consistent with transdifferentiation of choanocytes into archeocytes. Note the presence of nuclear fragments (fr) presumably resulting from phagocytosis that has occurred during de-differentiation [see Additional file 5: Figure S5]. Arrowheads in $\mathbf{E}$ and $\mathbf{F}$ show a labelled exopinacocyte and a sclerocyte, respectively, indicating these cell types can also be produced from de-differentiating choanocytes. Nuclei are stained with DAPI. Scale bars: $100 \mu \mathrm{m}$ (A, C), $10 \mu \mathrm{m}$ (B, D-F). DAPI, 4',6-diamidino-2-phenylindole.

\section{Conclusions}

Comparison of demosponge and eumetazoan development and body plans minimally provides insights into their last common ancestor, which appears to have existed some 800 million years ago [39]. Although the possibility that their last common ancestor possessed germ layers and these were lost in the demosponge lineage cannot be excluded, the lack of developmental commitment of larval and juvenile cell types in $A$. queenslandica and homology of internal layers across a demosponge and eumetazoans are consistent with eumetazoan germ layers and gastrulation evolving after the divergence of demosponge and eumetazoan lineages. Thus, we propose that ancestrally metazoans consisted of multiple cell layers whose fate was labile. The evolution of the gene regulatory network endowing progressive determination of cell layers resulted in the emergence of primary germ layers and mechanisms to 


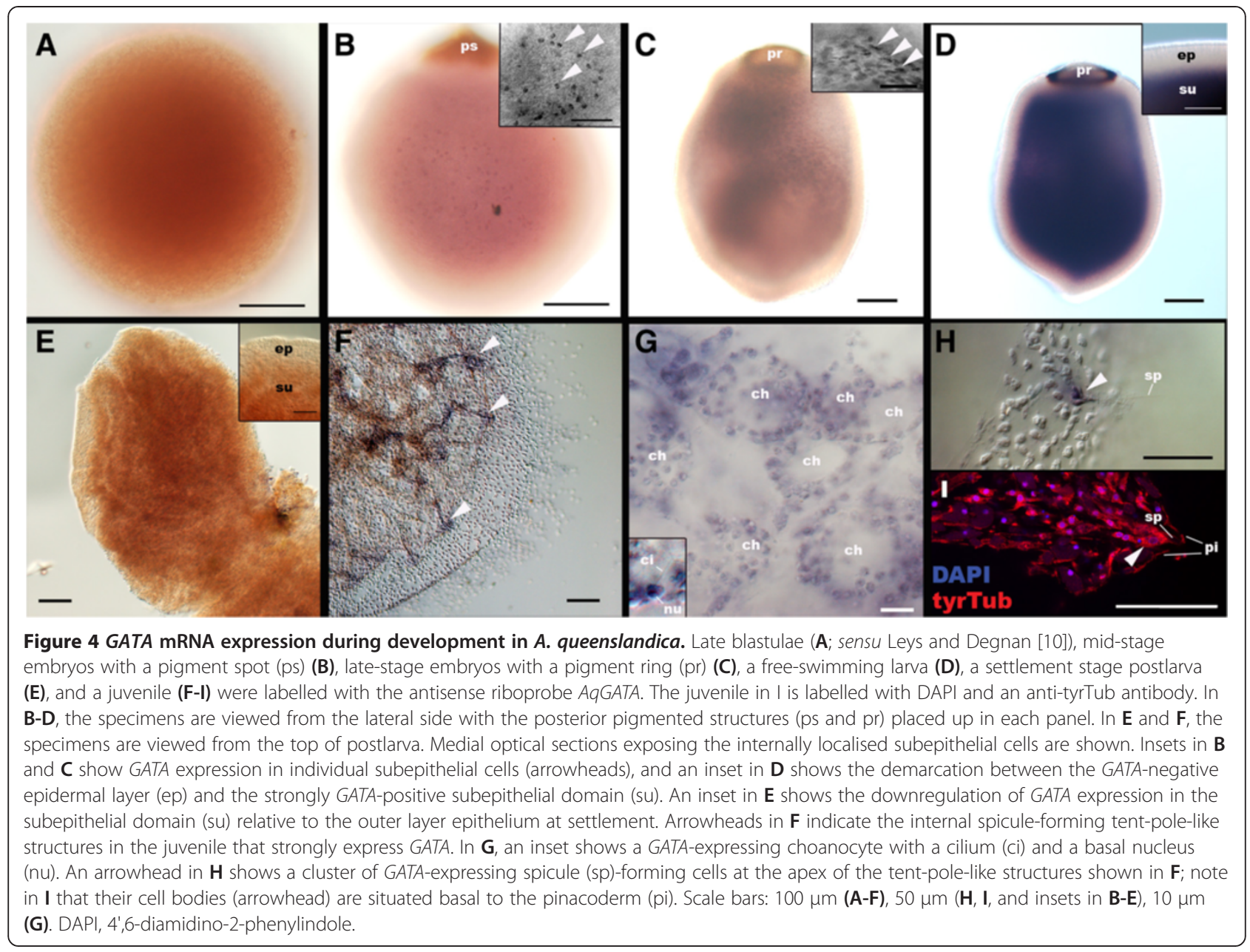

segregate these layers in eumetazoans (that is, gastrulation). The origin of the majority of genes that have conserved roles in eumetazoan gastrulation and germ layer determination evolved after the divergence of demosponge and eumetazoan lineages (for example, Fkh, Twist, Snail), giving credence to this supposition. The conserved expression of GATA in cells located inside the body of all metazoans, along with conserved differential expression of Wnt and TGF- $\beta$ ligands along demosponge and eumetazoan body axes [40], suggests that these genes were essential in providing critical positional information to cells in the first multicellular metazoans. These, along with eumetazoan-specific genes, later became essential components of the specification and determination of eumetazoan germ layers.

\section{Methods}

\section{Animal collection}

Adult A. queenslandica were collected at the Heron Island Research Station, Queensland, Australia. Larvae were collected upon release from adults and metamorphosing individuals were obtained by allowing larvae to settle and metamorphose on a plastic petri dish as previously described [41].

\section{Cell lineage tracing with CM-Dil}

The lipophilic tracer CM-DiI (Molecular Probes, C7000, Mulgrave, Victoria, AUS) was used to label ciliated epithelial cells in larvae and choanocytes in the juveniles. Larvae or juveniles were incubated in seawater with $10 \mu \mathrm{M}$ CM-DiI. To differentially label the plasma membrane of ciliated epithelial cells, larvae were incubated for 12 hours. Incubation for one hour was sufficient to differentially label the majority of choanocytes in juveniles. Little labelling occurred in internal cells in larvae or juveniles. The labelled specimens were rinsed in fresh seawater several times and then allowed to develop further. Specimens were fixed as described below at various stages of development. Nuclei were labelled with the fluorescent dye 4',6-diamidino-2-phenylindole (DAPI; 1:1,000, Molecular Probes), and the specimens were mounted in ProlongGold antifade reagent (Molecular Probes). 


\section{EdU pulse-and-chase experiments}

Larvae or juveniles were incubated in seawater containing $200 \mu \mathrm{M}$ of the thymidine analogue, EdU (Click-iT EdU AlexaFluor 488 cell proliferation kit, C10337, Molecular Probes), for 16 to 18 hours to label nuclei of proliferating larval archeocytes, and for three hours to label S-phase nuclei in the juveniles. Following washes in fresh seawater, the juveniles were immediately fixed. The larvae with labelled archeocytes were allowed to metamorphose into the juvenile stage, which were then fixed. Fixed specimens underwent immunohistochemistry as described below. Following the immunohistochemistry procedure, fluorescent labelling of incorporated EdU was conducted according to the manufacture's recommendations prior to DAPI labelling.

\section{Immunohistochemistry, TUNEL and confocal microscopy}

Larvae, postlarvae and juveniles were fixed as previously described [42]. Fixed specimens were rehydrated and washed in PBST (phosphate-buffered saline $+0.1 \%$ Tween20) and blocked with $1 \%$ bovine serum albumin (BSA) in PBST. Primary antibodies were incubated with the specimens in PBST. We used antibodies against phosphorylated histone H3 (phospho S10) (rabbit, 1:500, Abcam ab5176, Cambridge, MA, USA) and tyrosinated- $\partial$-tubulin (mouse, 1:500, Sigma T9028, Castle Hill, NSW, AUS) overnight at $4^{\circ} \mathrm{C}$. Following washes in PBST and blocking in 1\% BSA in PBST, secondary antibodies were incubated with the specimens; we used AlexaFluor 568 (anti-rabbit or -mouse. 1:200, Molecular Probes) and AlexaFluor 647 (anti-rabbit or -mouse, 1:200, Molecular Probes). Filamentous actin was labelled using AlexaFluor 488-conjugated phallacidin (1:25, Molecular Probes). Specimens were incubated with phallacidin together with secondary antibodies. After washes in PBST, the TUNEL assay was performed by using an In Situ Cell Death Detection Kit (TMR red cat no. 1684795, Roche, Indianapolis, IN, USA) following the manufacturer's protocol. Nuclei were labelled with DAPI and the specimens were mounted in ProlongGold antifade reagent as described above. Images were recorded using a Zeiss LSM 510 META Confocal Microscope and confocal stacks were viewed using ImageJ.

\section{Gene isolation and whole-mount in situ hybridization}

AqGATA gene fragments were amplified with gene specific primers, by using complimentary DNA from mixed developmental stages as a PCR template. Gene specific primers were as follows: F1, ATGGAGAAAGCAGACGACGCTA TGC; F2, CTACCAAGACAGTTTTGTGG; R1, CTACATC AATTGCTGTGGTTGCATGG and R2, ACTTTGACTT CTTTGACTCG. Primers F2 and R1 were used to generate a 776 base template for a riboprobe synthesis. Riboprobe synthesis and in situ hybridization were conducted as described previously [42]. An antisense digoxigenin-labeled riboprobe was hybridised at a final concentration of $1 \mathrm{ng} / \mu \mathrm{l}$.

\section{Transmission electron microscopy}

Free-swimming larvae were processed for transmission electron microscopy (TEM) by using the high-pressure freezing (HPF) technique. Procedures followed the previously established HPF protocol for shark retinal tissues [43] with the following modifications: $20 \%$ BSA in artificial seawater was used as a cryoprotectant instead of the teleost ringer $/ 0.7 \%$ agarose. In addition, $1 \% \mathrm{OsO}_{4}$ was used in the freeze substitution cocktail. Longitudinal ultrathin sections were prepared and analysed.

\section{Additional files}

\begin{abstract}
Additional file 1: Figure S1. Stages of development during
metamorphosis in A. queenslandica. A: Free-swimming larva stage; the (parenchymella) larva is egg-shaped and swims with the pigmented ring and associated bundles of long cilia directed posteriorly [10]. The anterior is down. In B-F, metamorphosing postlarvae are viewed from the top. B: Settlement stage. The anterior region of the larva is attached to the substrate, onto which the larva flattens. C: Mat-formation postlarval stage, cells of the metamorphosing postlarva migrate laterally on the substrate to form a mat-like structure. Note that former posterior-ring pigmentation (pi) is still visible but is disappearing. $\mathbf{D}$ : Chamber-formation postlarval stage, with developing canals (ca) lined by choanocyte chambers (ch) and endopinacoderm. Note the aquiferous system composed of canals lined by choanocytes and endopinacocytes becomes evident. E: Tent-pole-formation postlarval stage, the exopinacotes covering the outer surface of the metamorphosing postlarva are lifted upwards by formation of tent-pole-like structures consisting of vertically oriented clusters of spicules and associated cells. Arrowheads show the internal tent-pole-like structures, visible here as clustering of cells. F: Juvenile (rhagon) stage with an osculum (os), marking the establishment of the functional aquiferous system. Abbreviations: Ic long cilia; pr pigment ring; cc cuboidal cells. Scale bar: $100 \mu \mathrm{m}$ (A, inset in F), $1 \mathrm{~mm}$ (B-F).
\end{abstract}

Additional file 2: Figure S2. Apoptosis, loss of epithelial integrity and phagocytosis at early metamorphosis in A. queenslandica. A-C: Free-swimming larva. D-F: Settled postlarva, $\leq 7$ hours post-settlement. G: Settled postlarva, $\leq 12$ hours post-settlement. $\mathbf{H}$ : Mat-formation postlarval stage. DAPI is used to label DNA. In A, B, D, E and G, DNA fragmentation is detected by TUNEL assays (TUNEL). In C, F and G, specimens are labelled with an anti-Tyrosinated-Tubulin (tyrTub). A: A confocal longitudinal section through the larva; anterior is down. A boxed region in $\mathbf{A}$ is shown in $\mathbf{B}$; note that TUNEL-positive fragmented DNA (arrowheads) localises within the most anterior domain where cuboidal cells reside. C: A confocal section through the outer layer epithelium; note that the epithelium consists of a continuous sheet of cells with apical cilia (ci) and basal, typically anucleolated, nuclei (nu). D: Confocal medial sections viewed from the top of the metamorphosing postlarva. A single section through the outer layer epithelium in the boxed region in $\mathbf{D}$ is shown in $\mathbf{E}$. $\mathbf{F}$ : A confocal section showing the surface view of the distintegrating outer layer epithelium; note the breakup into smaller, tightly packed cells with anucleolated nuclei (the fragmented epithelium; fr). G: A confocal section of an outer-most region of the metamorphosing postlarva; note the lack of an epithelium, and the presence of phagocytised TUNEL-positive apoptotic bodies (arrowheads). H: A confocal section through an archeocyte in a mat-formation-stage postlarva labelled with DAPI and an anti-tyrTub, in which larval archeocytes and ciliated epidermal cells were simultaneously labelled (with EdU and CM-Dil, respectively) and followed through metamorphosis. Note intracellular CM-Dil-positive cellular fragments containing DAPI-positive nuclear materials (arrowhead), the presumptive apoptotic bodies, in an EdU-positive archeocyte of larval origin. Abbreviations: ep outer layer epithelium; icm inner cell mass. Scale bars: $100 \mu \mathrm{m}$ (A, D, E), $10 \mu \mathrm{m}$ (B, C, F-H).

Additional file 3: Figure S3. Larval ciliated epithelial cells lose cilia and transdifferentiate into choanocytes via an archeocyte intermediate during 
metamorphosis in A. queenslandica. Confocal sections through a flask cell (fc) at the free-swimming larva stage (A) and a transdifferentiating flask cell at the settlement stage labelled with CM-Dil (B). Note the loss of apical structures including the cilium (ci). C: Confocal sections viewed from the top at settlement, showing that many Dil-labelled cells are internalised (arrowheads). D: Confocal sections viewed from the top during metamorphosis; note that Dil-labelled cells have differentiated into archeocytes with characteristic large nucleoli (nu; inset magnified from the boxed cells). E: Confocal sections of individual archeocytes of the larval epidermal origin undergoing further differentiation at the chamber-formation stage; an arrowhead shows a differentiating choanocyte with a cilium (ci in inset) whose cell body has yet to complete cytokinesis within the mother archeocyte (ar). F: Confocal sections of a multi-nucleated archeocytechoanocyte intermediate at the chamber-formation stage. Differentiating choanocytes with cilia (ci) are evident (arrowheads). Abbreviations: ap apical non-ciliary process; ba basal process; co columnar epithelial cell. Scale bar: $10 \mu \mathrm{m}(\mathbf{A}, \mathbf{B}$, inset in $\mathbf{D}, \mathbf{E}, \mathbf{F}), 100 \mu \mathrm{m}(\mathbf{C}, \mathbf{D})$.

Additional file 4: Figure S4. EdU is incorporated into proliferating archeocytes in the inner cell mass in A. queenlandica larvae.

A, B: Confocal sections of the inner cell mass in a free-swimming larva pulse-labelled with EdU. Nuclei are labelled with DAPI. Arrowheads show EdU-positive nuclei. The boxed region in $\mathbf{B}$ is magnified in an inset. Note that the EdU-positive nuclei are 3.5 to $4.0 \mu \mathrm{m}$ in diameter and contain prominent nucleoli (nu), as observed in larval archeocytes in the inner cell mass in another demosponge Haliclona tubifera [44]. C: A transmission electron micrograph showing prominent nucleoli (nu) in nuclei of archeocytes (ar) in the inner cell mass within an A. queenslandica larva. Scale bar: $10 \mu \mathrm{m}$ (A, B), $5 \mu \mathrm{m}$ (C)

Additional file 5: Figure S5. Epithelial choanocytes can de-differentiate into archeocytes in A. queenslandica. Choanocytes in chambers (ch) in juveniles were labelled with CM-Dil as in Figure $3 \mathrm{~A}, \mathrm{~B}$ and descendants of the labelled cells in juveniles one day later are shown. Some choanocytes are seen to extend basal processes (ba) laterally, engulfing neighboring choanocytes (arrowheads in A, B). Some cells appear multinucleated (arrowheads in C), presumably as a result of phagocytic or fusion events. Abbreviations: ch choanocyte chamber; ci cilium. Scale bar: $10 \mu \mathrm{m}$.

Additional file 6: Figure S6. GATA sequence alignment and phylogenetic analyses. A: Partial GATA protein multiple sequence alignment showing the amino acid sites used for phylogenetic analyses. GATA genes encode zinc-finger transcription factors that bind to the (T/A (GATA) A/G) cis-regulatory motif [45] and are characterised by the presence of two zinc-finger domains ( $\mathrm{N}$-terminal ' $\mathrm{N}$-finger' and $\mathrm{C}$-terminal ' $\mathrm{C}$-finger'). The alignment encompasses $\mathrm{N}$-finger (underlined in red) and C-finger (underlined in blue) zinc-finger domains each encoding CXNC (boxed) $X_{17}$ CNXC (boxed). B: GATA maximum likelihood phylogeny rooted with GATA-type sequences. Amphimedon queenslandica sequence is highlighted in red. No clear GATA orthologues were identified outside Metazoa, and in the genome of the ctenophore Mnemiopsis leidyi [46]. C: Unrooted GATA maximum likelihood phylogeny. Sequence alignment and phylogenetic analyses were performed on the Geneious platform (v.5.1.7). Related sequences were retrieved via the protein BLAST search using the Amphimedon GATA-like sequence as queries, from GenBank at NCBI [47], Acropora digitifera genome (Version 1.1) portal at OIST [48], Mnemiopsis genome project portal at $\mathrm{NIH}$ [46] and Compagen at the University of Kiel (Oscarella sequences; [49]). Sequence IDs/accession numbers are shown with the name of each sequence in $\mathbf{B}$ and $\mathbf{C}$. Peptide sequences were aligned with MUSCLE (v3.7) [50] (default settings), and ambiguous regions were manually removed. Phylogenetic trees were reconstructed using the maximum likelihood method implemented in the PhyML program [51]. The WAG substitution model [52] was selected assuming an estimated proportion of invariant sites and four gammadistributed rate categories to account for rate heterogeneity across sites. The gamma shape parameter was estimated directly from the data. Reliability for internal branches of maximum likelihood trees was assessed using the bootstrapping method (100 bootstrap replicates). Support values are shown at each node except when lower than $50 \%$. The unit of the branch length is the number of substitutions per site.

Additional file 7: Table S1. A list of the presence or absence of $A$. queenslandica homologues (orthologues and gene family members) to cnidarian Nematostella vectensis endomesodermal genes (listed in [29]) based on previously published data $[28,53-58]$ and sequence similarity searches in NCBI databases (via BLASTp).

\section{Competing interests}

The authors declare that they have no competing interests.

\section{Authors' contributions}

$\mathrm{NN}$ and SS carried out the experiments and microscopy. NN and BMD conceived of the study and wrote the manuscript. All authors read and approved the final manuscript.

\section{Acknowledgements}

We thank the staff at the Heron Island Research Station for the use of the facilities, Dr. Kathryn Green at the Centre for Microscopy and Microanalysis at the University of Queensland for preparing EM sections, and Degnan lab members for constructive discussions. This research was supported by an Australian Research Council grant to BMD.

\section{Received: 20 January 2014 Accepted: 17 March 2014}

Published: 28 March 2014

\section{References}

1. Hyman LH: The Invertebrates: Protozoa Through Ctenophora, Volume 1. McGraw-Hill Book Company, Inc: New York and London; 1940.

2. Hall BK: Germ layers and the germ-layer theory revisited - primary and secondary germ layers, neural crest as a fourth germ layer, homology, and demise of the germ-layer theory. Evol Biol 1998, 30:121-186.

3. Martindale MQ: The evolution of metazoan axial properties. Nat Rev Genet 2005, 6:917-927.

4. Martindale $M Q$, Pang $K$, Finnerty JR: Investigating the origins of triploblasty: 'mesodermal' gene expression in a diploblastic animal, the sea anemone Nematostella vectensis (phylum, Cnidaria; class, Anthozoa). Development 2004, 131:2463-2474.

5. Nakanishi N, Renfer E, Technau U, Rentzsch F: Nervous systems of the sea anemone Nematostella vectensis are generated by ectoderm and endoderm and shaped by distinct mechanisms. Development 2012, 139:347-357.

6. Erpenbeck D, Worheide G: On the molecular phylogeny of sponges (Porifera). Zootaxa 2007, 1668:107-126.

7. Gazave E, Lapebie P, Ereskovsky AV, Vacelet J, Renard E, Cardenas P, Borchiellini C: No longer demospongiae: Homoscleromorpha formal nomination as a fourth class of porifera. Hydrobiologia 2012, 687:3-10.

8. Ereskovsky AV: The Comparative Embryology of Sponges. Dordrecht; New York: Springer; 2010.

9. Leys SP: Gastrulation in sponges. In Gastrulation, From Cells to Embryo. Edited by Stern CD. New York: Cold Spring Harbor Laboratory Press; 2004:23-31.

10. Leys SP, Degnan BM: Embryogenesis and metamorphosis in a haplosclerid demosponge: gastrulation and transdifferentiation of larval ciliated cells to choanocytes. Invert Biol 2002, 121:171-189.

11. Ereskovsky AV, Boury-Esnault N: Cleavage pattern in Oscarella species (Porifera, Demospongiae, Homoscleromorpha): transmission of maternal cells and symbiotic bacteria. J Nat Hist 2002, 36:1761-1775.

12. Boury-Esnault N, Efremova S, Bezac C, Vacelet J: Reproduction of a hexactinellid sponge: first description of gastrulation by cellular determination in the Porifera. Invert Reprod Dev 1999, 35:187-201.

13. Leys SP, Cheung E, Boury-Esnault N: Embryogenesis in the glass sponge Oopsacas minuta: formation of syncytia by fusion of blastomeres. Integr Comp Biol 2006, 46:104-117.

14. Gonobobleva E, Ereskovsky A: Polymorphism in free-swimming larvae of Halisarca dujardini (Demospongiae, Halisarcida). In Sponge Science in the New Millennium: Papers Contributed to the VI International Sponge Conference, Rapallo (Italy), 29 September-5 October 2002, Volume 68. Edited by Pansini M, Pronzato R, Bavestrello G, Manconi R. Genova, Italy: Boll Mus Ist Biol. Universitá di Genova; 2004:349-356.

15. Ereskovsky AV, Gonobobleva E: New data on embryonic development of Halisarca dujardini Johnson, 1842 (Demospongiae, Halisarcida). Zoosystema 2000, 22:355-368 
16. Leys SP, Eerkes-Medrano D: Gastrulation in calcareous sponges: in search of Haeckel's gastraea. Integr Comp Biol 2005, 45:342-351.

17. Simpson TL: Cell Biology of Sponges. New York: Springer-Verlag; 1984

18. Ereskovsky AV, Korotkova GP: The reasons of sponge sexual morphogenesis peculiarities. Berliner Geowiss Abh 1997, 20:25.

19. Bergquist PR, Green CR: Ultrastructural-study of settlement and metamorphosis in sponge larvae. Cahiers De Biologie Marine 1977, 18:289-302.

20. Meewis H: Contribution a l'étude de l'embryogenése de chalinulidae: Haliclona limbata. Ann Soc R Zool Belg 1939, 70:201-243.

21. Misevic GN, Burger MM: The molecular basis of species specific cell-cell recognition in marine sponges, and a study on organogenesis during metamorphosis. Prog Clin Biol Res B 1982, 85:193-209.

22. Misevic GN, Schlup V, Burger MM: Larval metamorphosis of Microciona prolifera: evidence against the reversal of layers. In New Perspectives in Sponge Biology. Edited by Rutzler K. Washington, DC: Smithsonian Institution Press; 1990:182-187.

23. Minchin EA: Note on the larva and the postlarval development of Leucosolenia variabilis, $H$. sp., with remarks on the development of other Asconidae. Proc R Soc Lond 1896, 60:42-52.

24. Amano S, Hori I: Metamorphosis of coeloblastula performed by multipotential larval flagellated cells in the calcareous sponge Leucosolenia laxa. Biol Bull 2001, 200:20-32.

25. Amano S, Hori I: Metamorphosis of calcareous sponges. 2. Cell rearrangement and differentiation in metamorphosis. Invert Reprod Dev 1993, 24:13-26.

26. Ereskovsky AV, Tokina DB, Bezac C, Boury-Esnault N: Metamorphosis of cinctoblastula larvae (Homoscleromorpha, Porifera). J Morphol 2007, 268:518-528.

27. Ereskovsky AV, Renard E, Borchiellini C: Cellular and molecular processes leading to embryo formation in sponges: evidences for high conservation of processes throughout animal evolution. Dev Genes Evol 2013, 223:5-22.

28. Srivastava M, Simakov O, Chapman J, Fahey B, Gauthier ME, Mitros T, Richards GS, Conaco C, Dacre M, Hellsten U, Larroux C, Putnam NH, Stanke M, Adamska M, Darling A, Degnan SM, Oakley TH, Plachetzki DC, Zhai YF, Adamski M, Calcino A, Cummins SF, Goodstein DM, Harris C, Jackson DJ, Leys SP, Shu SQ, Woodcroft BJ, Vervoort M, Kosik KS, et al: The Amphimedon queenslandica genome and the evolution of animal complexity. Nature 2010, 466:720-723.

29. Rottinger $E$, Dahlin $P$, Martindale MQ: A framework for the establishment of a cnidarian gene regulatory network for "endomesoderm" specification: the inputs of ss-catenin/TCF signaling. PLoS Genetics 2012, 8:e1003164.

30. Gillis WJ, Bowerman B, Schneider SQ: Ectoderm- and endomesodermspecific GATA transcription factors in the marine annelid Platynereis dumerilli. Evol Dev 2007, 9:39-50.

31. Chiodin M, Borve A, Berezikov E, Ladurner P, Martinez P, Hejnol A: Mesodermal gene expression in the acoel Isodiametra pulchra indicates a low number of mesodermal cell types and the endomesodermal origin of the gonads. PLOS One 2013, 8:e55499.

32. Boyle MJ, Seaver EC: Developmental expression of foxA and gata genes during gut formation in the polychaete annelid, Capitella sp I. Evol Dev 2008, 10:89-105.

33. Srivastava M, Begovic E, Chapman J, Putnam NH, Hellsten U, Kawashima T, Kuo A, Mitros T, Salamov A, Carpenter ML, Signorovitch AY, Moreno MA, Kamm K, Grimwood J, Schmutz J, Shapiro H, Grigoriev IV, Buss LW, Schierwater B, Dellaporta SL, Rokhsar DS: The Trichoplax genome and the nature of placozoans. Nature 2008, 454:955-960.

34. Ryan JF, Pang K, Schnitzler CE, Nguyen AD, Moreland RT, Simmons DK, Koch BJ, Francis WR, Havlak P, Comparative Sequencing Program NISC, Smith SA, Putnam NH, Haddock SH, Dunn CW, Wolfsberg TG, Mullikin JC, Martindale $\mathrm{MQ}$, Baxevanis AD: The genome of the ctenophore Mnemiopsis leidyi and its implications for cell type evolution. Science 2013, 342:1242592.

35. Riesgo A, Farrar N, Windsor PJ, Giribet G, Leys SP: The analysis of eight transcriptomes from all poriferan classes reveals surprising genetic complexity in sponges. Mol Biol Evol 2014. doi:10.1093/molbev/msu057.

36. Yuan D, Nakanishi N, Jacobs DK, Hartenstein V: Embryonic development and metamorphosis of the scyphozoan Aurelia (Cnidaria, Scyphozoa). Dev Genes Evol 2008, 218:525-539.

37. Weis $\vee$, Buss $L$ : Ultrastructure of metamorphosis in Hydractinia echinata. Postilla 1987, 199:1-20
38. Sperling EA, Peterson KJ, Pisani D: Phylogenetic-signal dissection of nuclear housekeeping genes supports the paraphyly of sponges and the monophyly of Eumetazoa. Mol Biol Evol 2009, 26:2261-2274.

39. Erwin DH, Laflamme M, Tweedt SM, Sperling EA, Pisani D, Peterson KJ: The Cambrian conundrum: early divergence and later ecological success in the early history of animals. Science 2011, 334:1091-1097.

40. Adamska M, Degnan SM, Green KM, Adamski M, Craigie A, Larroux C, Degnan BM: Wnt and TGF-beta expression in the sponge Amphimedon queenslandica and the origin of metazoan embryonic patterning. PLoS One 2007, 2:e1031.

41. Leys SP, Larroux C, Gauthier M, Adamska M, Fahey B, Richards GS, Degnan SM, Degnan BM: Isolation of Amphimedon developmental material. CSH protocols 2008, 2008: pdb.prot5095.

42. Larroux C, Fahey B, Liubicich D, Hinman VF, Gauthier M, Gongora M, Green K, Worheide G, Leys SP, Degnan BM: Developmental expression of transcription factor genes in a demosponge: insights into the origin of metazoan multicellularity. Evol Dev 2006, 8:150-173.

43. Harahush BK, Green K, Webb R, Hart NS, Collin SP: Optimal preservation of the shark retina for ultrastructural analysis: an assessment of chemical, microwave, and high-pressure freezing fixation techniques. Microsc Res Tech 2012, 75:1218-1228.

44. Woollacott RM: Structure and swimming behavior of the larva of Haliclona tubifera (Porifera, Demospongiae). J Morphol 1993, 218:301-321.

45. Felsenfeld G, Evans T, Reitman M: An erythrocyte-specific DNA-binding factor recognizes a regulatory sequence common to all chicken globin genes. Proc Natl Acad Sci U S A 1988, 85:5976-5980.

46. Mnemiopsis Genome Project Portal. [http://research.nhgri.nih.gov/mnemiopsis/]

47. Basic Local Alignment Search Tool. [http://blast.ncbi.nlm.nih.gov/Blast.cgi]

48. Acropora digitifera Genome (Ver 1.1). [http://marinegenomics.oist.jp/genomes/ viewer?project_id=3\&current_assembly_version=oist_v1.1]

49. Compagen: A comparative genomics platform for early branching Metazoa. [http://compagen.zoologie.uni-kiel.de/]

50. Edgar RC: MUSCLE: multiple sequence alignment with high accuracy and high throughput. Nucleic Acids Res 2004, 32:1792-1797.

51. Gascuel O, Guindon S: A simple, fast, and accurate algorithm to estimate large phylogenies by maximum likelihood. Syst Biol 2003, 52:696-704.

52. Whelan S, Goldman N: A general empirical model of protein evolution derived from multiple protein families using a maximum-likelihood approach. Mol Biol Evol 2001, 18:691-699.

53. Simionato E, Ledent V, Richards G, Thomas-Chollier M, Kerner P, Coornaert $D$, Degnan BM, Vervoort M: Origin and diversification of the basic helix-loop-helix gene family in metazoans: insights from comparative genomics. BMC Evol Biol 2007, 7:33.

54. Larroux C, Luke GN, Koopman P, Rokhsar DS, Shimeld SM, Degnan BM: Genesis and expansion of metazoan transcription factor gene classes. Mol Biol Evol 2008, 25:980-996.

55. Larroux C, Fahey B, Degnan SM, Adamski M, Rokhsar DS, Degnan BM: The NK homeobox gene cluster predates the origin of Hox genes. Curr Biol 2007, 17:706-710.

56. Ryan JF, Mazza ME, Pang K, Matus DQ, Baxevanis AD, Martindale MQ Finnerty JR: Pre-bilaterian origins of the Hox cluster and the Hox code: evidence from the sea anemone, Nematostella vectensis. PLoS One 2007, 2:e153.

57. Kerner P, Degnan SM, Marchand L, Degnan BM, Vervoort M: Evolution of RNA-binding proteins in animals: insights from genome-wide analysis in the sponge Amphimedon queenslandica. Mol Biol Evol 2011, 28:2289-2303.

58. Adamska M, Larroux C, Adamski M, Green K, Lovas E, Koop D, Richards GS, Zwafink C, Degnan BM: Structure and expression of conserved Wnt pathway components in the demosponge Amphimedon queenslandica. Evol Dev 2010, 12:494-518.

\section{doi:10.1186/1741-7007-12-26}

Cite this article as: Nakanishi et al:: Evolutionary origin of gastrulation: insights from sponge development. BMC Biology 2014 12:26. 